\title{
Zebrafish as a Model Organism to Study Nanomaterial Toxicity Jaison Jeevanandam ${ }^{\mathrm{a}}$, Yen San Chan ${ }^{\mathrm{a}}$, Michael K. Danquah ${ }^{\mathrm{b}}$ \\ ${ }^{a}$ Department of Chemical Engineering, Faculty of Engineering and Science, Curtin University CDT 250, 98009, Miri, Sarawak, Malaysia \\ ${ }^{b}$ Chemical Engineering Department, University of Tennessee, Chattanooga, TN 37403, Unites States
}

\begin{abstract}
Recent developments in nanotechnology has increased the market value of nanoproducts in various industries. This has increased concerns associated with potential toxicity of nanoproducts to humans and the environments. Even though, green and biosynthesized nanoparticles are considered to be less toxic than chemically synthesized nanoparticles, they still possess some level of toxicity. Conventional toxicity assessments via human cells, live animals such as rat, frog or rabbit have several drawbacks including ethical issue and challenges involving the maintenance and development of cell cultures. Zebrafish (Danio rerio) is a transparent vertebrate fish that can reproduce rapidly. Its larvae develop in 5 days up to $3-5 \mathrm{~cm}$ long. It also possesses about $69 \%$ similar genetic profile, molecular mechanism, cell development and organ physiology as humans. Hence, it has the potential to be utilized as an alternative to humans or live animal models for initial drug screening and toxicity tests. European Union, USFDA and ICH have approved the use of zebrafish for toxicological evaluation of pharmaceutical products including nanomedicines. The article presents for the potential of zebrafish in preclinical evaluation of the toxicity of nanomaterials. It also discusses other potential applications, including medical imaging and environmental toxicity.
\end{abstract}

Keywords:

Zebrafish;

Nanomaterials;

Toxicity Test;

Preclinical Trials

Model Organism

Article History:

Received: 06 April 2019

Accepted: 30 May 2019

\section{1- Introduction}

Zebrafish (Danio rerio) are a tropical teleost, diploid vertebrate fish which is $3-5 \mathrm{~cm}$ long and reproduces rapidly [1]. They are small in size, transparent, simple to handle, species with elevated fecundity, continuous reproduction and rapid embryogenesis [2]. Due to its size, zebrafish is easy to maintain in small spaces, and their rapid reproduction capability helps in large scale studies. The embryo and larva of zebrafish, called fry, are transparent and develop in 5 days. Hence, it is possible to grow zebrafish in a petridish [3]. Several studies have reported that zebrafish can be used as a potential in vivo model to analyze the efficiency and genetic toxicity of drugs. Each stage of the zebrafish growth cycle is advantageous in certain aspects for toxicity studies especially genotoxicity [4]. Embryos of zebrafish are beneficial in fish embryo assay as they are pain-free in vivo tests, and this could be an alternative for animal experiments [5]. Shi et al. (2007) demonstrated that zebrafish embryos can be used to identify the developmental toxicity and variations in gene expression after exposing it with a persistent organic pollutant called perflurooctanesulfonate (PFOS) [6]. The transparent larvae of zebrafish makes them excellent for fluorescent protein visualization, identifying neutrophils after $48 \mathrm{~h}$ of fertilization and determining the existence of innate immune system isolated from adaptive systems [7, 8]. Renshaw et al. (2006) utilized transgenic zebrafish larvae as an in vivo model to analyze genetic inflammatory response by expressing green fluorescent protein under the neutrophil-specific myeloperoxidase promoter [9]. Similarly, adult

\footnotetext{
* CONTACT: Jaison.jeevanandam@ postgrad.curtin.edu.my
}

DOI: http://dx.doi.org/10.28991/esj-2019-01182

(C) 2019 by the authors. Licensee ESJ, Italy. This is an open access article under the terms and conditions of the Creative Commons Attribution (CC-BY) license (https://creativecommons.org/licenses/by/4.0/). 
zebrafish are considered as an exclusive model to analyze vertebrate development, especially immune system progression. Neely et al. (2002) demonstrated the effect and mechanism of bacterial infection caused by gram-positive Streptococcus iniae (a pathogen of fish and human), using an adult zebrafish [10].

Nanoparticles have emerged as novel small sized particles for the development of next generation materials with exclusive properties for various applications including electronics and medicine [11,12]. There are a wide variety of synthesis methods that are available for the generation of nanoparticles and the selection of the synthesis approach is crucial to obtain the desired characteristics of nanoparticles for specific applications [11, 13]. Chemical synthesis approaches are used to generate large quantities of nanosized particles. However, the chemical and reagents used for these synthesis approaches are identified to be toxic to human and the environment. Hence, chemical-based approaches are unsuitable for environmental and biomedical applications [14, 15]. Green and biosynthesis approaches have been introduced to develop nanoparticles that are biocompatible with reduced toxicity for biomedical and drug delivery applications $[16,17]$. Thus, toxicological analysis plays a significant role in validating the use of nanoparticles for medicine, bioimaging and environmental applications.

In vitro, in silico and in vivo analysis are the conventional methods to evaluate the toxicity of nanoparticles [18]. In vitro analysis includes cell lines and microbes to evaluate the toxicity of nanoparticles [19], whereas, in silico analysis involves computational simulations and models for toxicity evaluations [20]. Both methods are beneficial for preliminary toxicological assessment of nanoparticles for biomedical, environmental and antimicrobial applications [21]. However, in vivo models including live animal models are used for the validation of toxic reactions of nanoparticles. Rat, mouse, monkey, frog and cockroach are the animal models that are used as traditional in vivo toxicity models [22]. Even though, they provide useful results with clinical relevance, difficulty in growth and maintenance, tedious ethical clearance processes and practical/emotional difficulties in sacrificing large number of animals remain as drawbacks [23]. Thus, zebrafish is proposed as an alternative model for in vivo toxicological evaluation of nanoparticles. The genetic profile, cell development and organ physiology of zebrafish has $69 \%$ resemblance to humans. They also possess organs and tissues with analogues structures and functions, like humans. This includes the heart, kidney, liver, pancreas, intestinal tract and brain [24]. This article evaluates the efficiency of zebrafish as an effective in vivo animal model for the assessment of nanoparticle toxicity. In addition, the mechanism of nanoparticle toxicity in zebrafish and future perspectives on nanotoxicity analysis with zebrafish are also discussed.

\section{2- Zebrafish as an in Vivo Animal Model}

Zebrafish is proposed to be useful as an alternative to human cells in preclinical trials, toxicity analysis of medicines, complicated nerves and brain related drug development, and medical imaging applications according to Figure 1.

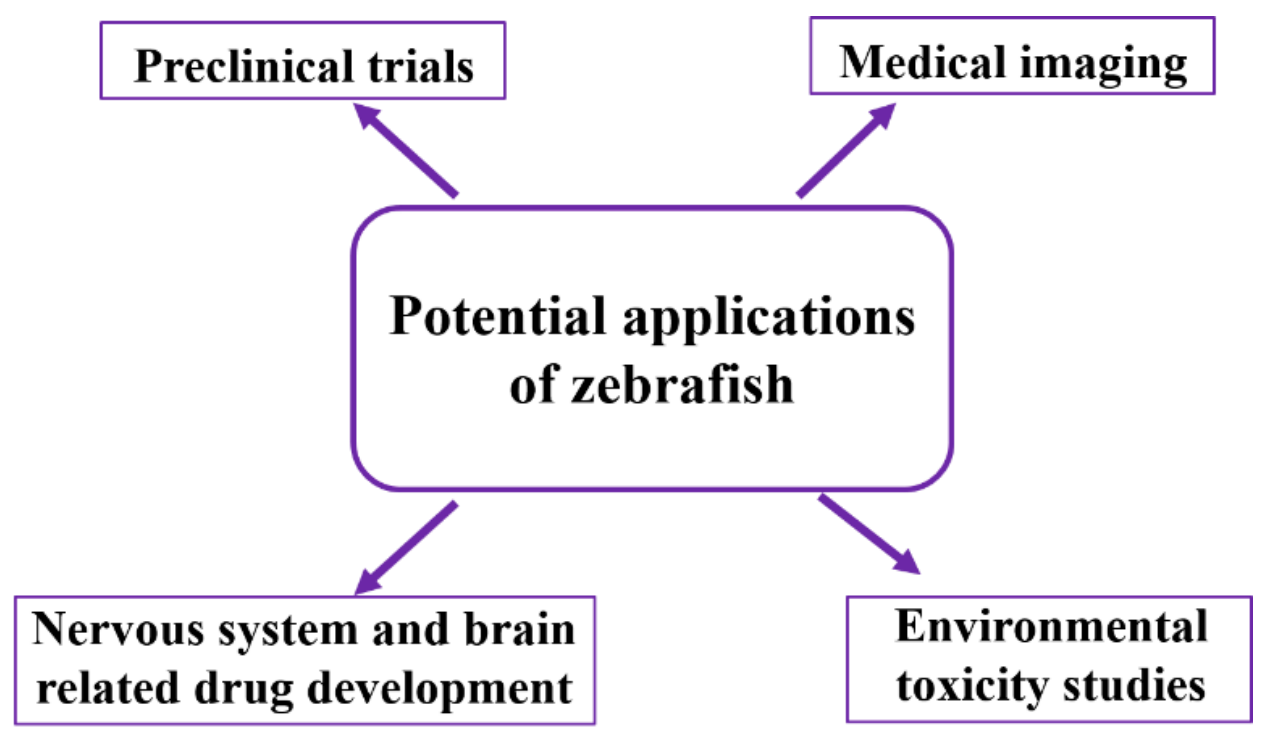

Figure 1. Potential applications of zebrafish as an animal model.

\section{2-1- Preclinical Trials}

The life stages of zebrafishes are widely used as an efficient animal model to evaluate drug effectiveness and screening analysis. In recent times, it has been used as an alternate vertebrate animal model for the discovery of phenotype-based drugs. Zebrafish possesses advantages such as accessible broad biology range and early toxicity insight [25]. The use of cell lines to screen drugs causes a drawback as it can screen only cell-autonomous phenotypes. The use of zebrafish offers evaluation with live animal models, having $\mathrm{s}$ a distinct repertoire, similar to the biological processes 
in humans with a completely integrated system of vertebrate organs. Thus, zebrafish can be used for preclinical drug screening analysis to evaluate and analyze the potency of drugs for the treatment of pain, tumor metastasis, gut motility, sedation and vascular tone [26]. In addition, cell line studies do not provide adequate insights into the pharmacokinetic and pharmacodynamic characteristics of drugs such as absorption, distribution, metabolism, excretion and toxicity, whereas zebrafish has demonstrated the capacity to provide pharmacokinetic and pharmacodynamic characteristics in live models with functional livers, kidneys and blood brain barriers [27]. Parng et al. (2002) showed that zebrafish can be a potential alternative animal model to replace existing cell line based on preclinical methods to evaluate the toxicity, angiogenesis and apoptosis of drugs. About 18 chemicals were analysed to obtain lethal concentration (LC50) levels in comparison with mice models. The results revealed that zebrafish is highly beneficial in preclinical studies of drugs than mice [28]. In recent times, Wertman et al. (2016) reported that zebrafish can be utilized as a xenograft platform for novel cancer model and as a preclinical screening tool [29]. In addition, Lenard et al. (2016) showed that zebrafish are beneficial as a live model to evaluate erythroid lineage toxicity and regeneration [30]. Zebrafish has also been demonstrated to be useful for preclinical evaluation and screening of drugs for the treatment of diseases such as cancer [31], Amyotrophic lateral sclerosis (ALS) [32], neurodegenerative diseases [33], Alzheimer's disease [34], cardiovascular and metabolic diseases [35], tuberculosis [36], Huntington's disease [37], muscle diseases [38], polycystic kidney diseases [39] and Parkinson's disease [40].

\section{2-2- Medical Imaging}

Apart from preclinical trials, zebrafish are also used as a live animal model to evaluate the efficiency of medical imaging tools. X-ray based imaging techniques require pre-evaluation before utilization for human organ imaging, as $\mathrm{X}$-ray exposure can harm cells due to their high energy [41]. Thus, zebrafish are used as a live animal model to evaluate and optimize the $\mathrm{x}$-ray energy intensity for human imaging applications. Zebrafish has been used to evaluate the efficiency of Synchrotron X-ray fluorescence (SXRF) microtomography to obtain three-dimensional images of transition metals in live animal models [42]. Likewise, $\mathrm{x}$-ray and light optics were used for whole-animal imaging, gene function and the zebrafish phenome project [43]. Synchrotron X-ray micro computed tomography has been used to obtain the entire body image of a hypercholesterolemic female zebrafish, as a test to demonstrate the application of the imaging technique to monitor cholesterol levels in live animal models [44]. In addition, it has been revealed that zebrafish are useful as live models to evaluate the efficiency of x-ray phase-contrast tomography for high spatial resolution muscle imaging [45], high resolution-ray vascular network imaging [46] and elemental as well as chemical specific x-ray fluorescence imaging of biological systems [47]. Laboratory-based x-ray NanoCT has been used to study the morphology of embryos [48], multifunctional green fluorescence carbon dots as fluorescent probe to detect mercury [49] and liquid metal jet x-ray sources for high resolution biomedical imaging [50] based on zebrafish as an in vivo model. Other than X-ray related imaging methods, zebrafish have also been used to examine the efficiency of twophoton time lapse live imaging of lymphatic development [51], high frequency ultrasound (45-75 Mhz) for in vivo cardiac imaging [52], optical projection tomography for in vivo label-free three dimensional vasculature imaging [53], and optoacoustic tomography for non-invasive whole-body imaging technique [54]. Zebrafishes also used as a significant in vivo model to evaluate the medical imaging methods of accelerated optical projection tomography [55], four dimensional light sheet to image cardiac development [56], functional optoacoustic neuro-tomography of calcium fluxes in adult brain [57] and carbon quantum dots based fluorescence imaging methods [58].

\section{2-3- Nervous System and Brain Related Drug Development}

Numerous studies have emphasized that zebrafish can be useful as a live animal model for the evaluation of neurotoxicity and for the determination of drug dosage to treat patients with nervous disorders [59]. Zebrafish also serve as an excellent in vivo model for rapid behavior-based identification of neuroactive small molecules as potential drugs for neurodegenerative disease treatment [60]. Zebrafish contain similar neuroanatomy and neurochemistry of the central nervous system in humans, and this is beneficial to evaluate the efficiency of drugs in treating neuropsychiatric diseases [61]. They also contain second presenilin gene, which is similar to the gene present in human Alzheimer's disease [62]. Thus, zebrafish are recently used as a tool for research and preparation of drugs for Alzheimer's disease [63]. Quercetin and rutin were injected into zebrafish for drug efficacy studies, and the result revealed that these drugs are beneficial in preventing scopolamine-induced memory impairment [64]. Further, zebrafish are widely used to evaluate and monitor neurogenic phenotype [65], modulatory neurotransmitter systems and behavior [66], neurogenic phenotype [65] and DJ1 expression [67]. Zebrafish was used to evaluate the neuroprotective ability of lanthionine ketimine-5-ethyl ester against okadaic acid-induced Alzheimer's disease [68]. Also, zebrafish have been used to effectively study accelerated brain aging towards transcriptional inversion [69], efficiency of GSK3 $\beta$ inhibitor, TDZD-8 [70], protective effects of quercetin [71] and gender specific expression changes in aging and Alzheimer's disease [72]. Zebrafish have been employed in research on Parkinson's disease [73, 74], Huntington's disease [75, 76] and amyotrophic lateral sclerosis [77, 78] to evaluate, monitor and study the disease physiology as well as drug efficacy. Zebrafish are considered an effective live animal model to screen drugs for brain cancer [79, 80] and ischemic stroke [81, 82]. All these studies provide supporting data on the efficacy of zebrafish for in vivo evaluation and study of nerve and brain related diseases. 


\section{2-4- Environmental Toxicity Studies}

Apart from medical and pharmaceutical evaluations, zebrafish are extensively used in environmental risk assessment studies. It has been reported that zebrafish, specifically embryos, can be used, not only to study acute toxicity, but also to evaluate teratogenicity, identifying mode of action, toxicokinetics, toxicodynamics and prediction of adverse and long term effects of environmental toxicity, especially toxicity due to heavy metals exposure towards an organism [83]. Further, zebrafish are widely explored as a potential animal model for the evaluation of environmental toxicity of chemicals via fish embryo toxicity test [84]. They also served as an effective vertebrate model for investigating chemical toxicity [85], dioxin developmental toxicity [86], structure-activity relationship assessment of perfluorinated chemicals [87], and proteotoxicity and embryotoxicity carrier solvents towards the environment [88]. In addition, the toxicity of heavy metals such as mercury, copper, nickel, lead and cobalt towards embryos and larvae of organisms have been evaluated using zebrafish [89]. Similarly, the toxicity of lead and tin based perovskite solar cells [90], chlorpyrifos, nickel chloride [91], and cadmium induced deformities in aquatic animals [92] have been evaluated using zebrafish. In recent times, zebrafish are used to investigate the time-response characteristics and potential biomarker identification of heavy metals [93], influence of microplastics on the accumulation and chronic toxicity of cadmium [94], and elevation of heavy metal toxicity via polyaspartic acid [95]. Thus, it is noteworthy that toxicity analysis of chemicals using zebrafish models will be an essential test in future to evaluate their toxicity mechanisms.

\section{3- Zebrafish for Nanotoxicity Evaluation}

The use of zebrafish as a tool to evaluate the toxicity of nanoparticles towards living organisms has received significant interests. The toxicity of nanoparticles has become a topic of interest since the synthesis of most nanoparticles involves the use of toxic chemicals. For example, gold nanoparticles are synthesized using cetyl trimethyl ammonium bromide (CTAB), which is proven to be toxic to human cells and tissues [96]. Several methods have been employed to evaluate the toxicity of nanoparticles in vitro using microbes [97], cell lines [98] as well as in vivo methods using animal models such as mice [99], cockroach [100] and drosophila [101]. However, each method poses some drawbacks that may be resolved using a combination of models. In vitro toxicological analysis of nanoparticles is not sufficient to evaluate their toxicity in animals as they represent only a specific type of cells which may differ in animals due to the combination and accumulation of nanoparticles in their metabolic pathway [102]. Thus, it is necessary to use in vivo toxicity analysis via live animals. Major challenges associated with the use of live animal models include the need for ethical clearance, animal safety clearance, hands-on training in animal dissection and errors in toxicology result and restrictions in the number of animals that can be used [103]. Thus, zebrafish, which is a simple fish model with easy approval process for ethical clearance and animal safety, are used as an effective tool for evaluating the toxicity of nanoparticles in live animal models [104]. In addition, zebrafish can turn into a fertile adult in two or three months and can lay about 200 eggs [105]. This high reproductive ability and fertility along with simple maintenance procedures contributes to the positive attributes of zebrafish for use in nanotoxicological analysis. The toxicity of almost all types of nanoparticles including metal [106], metal oxide [107], carbon [108], polymer nanoparticles [109], nanocomposites [110], zero-dimensional [58], one-dimensional [111], two-dimensional [112] and three-dimensional nanoparticles [113] have been evaluated directly using zebrafish with promising results as shown in Table 1. Zebrafish are also used to evaluate the genotoxicity of nanoparticles, as it is easy to analyze the genome of zebrafish compared to larger animals [114]. Zebrafish are emerging as an effective replacement of mice models for toxicological evaluation and screening of nanoparticles.

Table 1. Use of zebrafish for toxicological evaluation of nanoparticles.

\begin{tabular}{ccc}
\hline Nanoparticles & Description & Reference \\
\hline Gold & Metal nanoparticles & \\
Silver & Adult and embryo & {$[115,116]$} \\
Copper & Embryo and adult & {$[117,118]$} \\
Selenium & Embryo & {$[119]$} \\
Platinum & Embryo & {$[120]$} \\
Aluminium & Embryo & {$[122]$} \\
\hline Zinc oxide & Adult & {$[123]$} \\
Copper oxide & Metal oxide nanoparticles & {$[124]$}
\end{tabular}




\begin{tabular}{|c|c|c|}
\hline Titanium dioxide & Embryo & [125] \\
\hline Cerium dioxide & Early life stages & [126] \\
\hline Magnesium oxide & Embryo and larval stages & [127] \\
\hline Silica & Embryo & [128] \\
\hline Iron oxide & Life stages & [129] \\
\hline \multicolumn{3}{|c|}{ Carbon-based nanoparticles } \\
\hline Multiwalled carbon nanotubes & Embryo & [130] \\
\hline Single wall carbon nanotubes & Embryo & [131] \\
\hline Graphene quantum dot & Embryo & [132] \\
\hline Carbon dots & Embryo & [133] \\
\hline Pristine graphene & Embryo & [134] \\
\hline Graphene oxide & Larvae & {$[135]$} \\
\hline Fullerene & Embryo and adult & {$[136,137]$} \\
\hline \multicolumn{3}{|c|}{ Polymer nanoparticles } \\
\hline Chitosan & Embryo & [138] \\
\hline Hydroxy apatite & Embryo & [139] \\
\hline Poly (lactic-co-glycolic acid) (PLGA) & Life stages and adult & [140] \\
\hline $\begin{array}{l}\text { Anionic and cationic polyamidoamine } \\
\text { (PAMAM) and poly (propylene imine) (PPI) }\end{array}$ & Embryo & [141] \\
\hline Bismuth-asparagine & Embryo & [142] \\
\hline Redox polymer & Embryo & {$[143]$} \\
\hline Iron chelator starch-deferoxamine & Embryo & [144] \\
\hline \multicolumn{3}{|c|}{ Nanocomposites } \\
\hline Graphene oxide-titanium dioxide & Embryo and larvae & [145] \\
\hline Chitosan-zinc oxide & Early stages of zebrafish & [146] \\
\hline Starch-magnetite & Adult & [147] \\
\hline Titanium dioxide-multiwalled carbon nanotube & Embryo & [148] \\
\hline Gold-gadolinium doped-carbon quantum dot & Embryo & [149] \\
\hline Titanium dioxide-chondroitin-4-sulfate & Embryo and life stages & [150] \\
\hline
\end{tabular}

\section{4- Future Perspective}

It is noteworthy from Table 1 that the embryo of zebrafish is widely used for the toxicity evaluation of nanoparticles to determine the effects of nanoparticles on reproduction during development stages. The larval, life stages and adult of zebrafish are extensively used to examine the genotoxicity of nanoparticles towards live animal models. In future, zebrafish can be developed into potential live animal model for in vivo screening and evaluation of nanoparticles toward various diseases. There are several reports in recent times which demonstrate the capacity of zebrafish for screening and evaluating nanomedicines for the treatment of kidney diseases [151], cancer [152], diabetes [153], T-cell acute lymphoblastic leukemia [154] and human aging related diseases [155]. Improvements in the zebrafish embryo tests will further benefit researchers to examine the toxicity of nanoparticles in live embryos for various biomedical applications [156]. Zebrafish are considered as a promising alternative for nanotoxicological evaluation and drug discovery analysis to screen, evaluate and obtain benign nanomaterials for various applications. 


\section{5- Conclusion}

The main objective of this article is to provide an account to support the use of zebrafish for toxicological assessment of nanoparticles. Strict regulations and further developments in zebrafish related nanotoxicological assays such as fish embryo tests will enhance the efficacy of zebrafish in toxicity studies. Zebrafish can also be used in preclinical studies of nanomedicines due to their advantages over conventional in vitro and in vivo studies. However, there exists certain drawbacks, such as uncertainty in the result and misinterpretation of genotoxicity as well as classification of specific life stages in zebrafish, which should be addressed via specific physiological studies to enable a wide-spread application of zebrafish in toxicological analysis. Thus, zebrafish can become an alternative to conventional in vitro and in vivo models used in toxicological studies to facilitate the application of nanoparticles in biomedical and environmental applications.

\section{6- Conflict of Interest}

The authors declare no conflict of interest.

\section{7- Ethical Approval}

This work did not involve the use of animals and therefore ethical approval was not necessarily required.

\section{8- References}

[1] Grunwald, David Jonah, and Judith S. Eisen. "Headwaters of the Zebrafish — Emergence of a New Model Vertebrate." Nature Reviews Genetics 3, no. 9 (September 2002): 717-724. doi:10.1038/nrg892.

[2] Voelker, Doris, Christoph Vess, Michaela Tillmann, Roland Nagel, Georg W. Otto, Robert Geisler, Kristin Schirmer, and Stefan Scholz. "Differential Gene Expression as a Toxicant-Sensitive Endpoint in Zebrafish Embryos and Larvae." Aquatic Toxicology 81, no. 4 (March 2007): 355-364. doi:10.1016/j.aquatox.2006.12.013.

[3] Guo, S. "Linking Genes to Brain, Behavior and Neurological Diseases: What Can We Learn from Zebrafish?" Genes, Brain and Behavior 3, no. 2 (April 2004): 63-74. doi:10.1046/j.1601-183x.2003.00053.x.

[4] Goldsmith, J. R., and Christian Jobin. "Think Small: Zebrafish as a Model System of Human Pathology.” Journal of Biomedicine and Biotechnology 2012 (2012): 1-12. doi:10.1155/2012/817341.

[5] Rizzo, Larissa Y., Susanne K. Golombek, Marianne E. Mertens, Yu Pan, Dominic Laaf, Janine Broda, Jabadurai Jayapaul, et al. “In Vivo Nanotoxicity Testing Using the Zebrafish Embryo Assay.” Journal of Materials Chemistry B 1, no. 32 (2013): 3918. doi:10.1039/c3tb20528b.

[6] Shi, Xiongjie, Yongbing Du, Paul K.S. Lam, Rudolf S.S. Wu, and Bingsheng Zhou. "Developmental Toxicity and Alteration of Gene Expression in Zebrafish Embryos Exposed to PFOS.” Toxicology and Applied Pharmacology 230, no. 1 (July 2008): 2332. doi:10.1016/j.taap.2008.01.043.

[7] Lieschke, G. J. "Morphologic and Functional Characterization of Granulocytes and Macrophages in Embryonic and Adult Zebrafish.” Blood 98, no. 10 (November 15, 2001): 3087-3096. doi:10.1182/blood.v98.10.3087.

[8] Trede, Nikolaus S, David M Langenau, David Traver, A.Thomas Look, and Leonard I Zon. "The Use of Zebrafish to Understand Immunity.” Immunity 20, no. 4 (April 2004): 367-379. doi:10.1016/s1074-7613(04)00084-6.

[9] Renshaw, S. A., C. A. Loynes, D. M.I. Trushell, S. Elworthy, P. W. Ingham, and M. K.B. Whyte. “A Transgenic Zebrafish Model of Neutrophilic Inflammation.” Blood 108, no. 13 (December 15, 2006): 3976-3978. doi:10.1182/blood-2006-05-024075.

[10] Neely, M. N., J. D. Pfeifer, and M. Caparon. “Streptococcus-Zebrafish Model of Bacterial Pathogenesis.” Infection and Immunity 70, no. 7 (July 1, 2002): 3904-3914. doi:10.1128/iai.70.7.3904-3914.2002.

[11] Jeevanandam, Jaison, Ahmed Barhoum, Yen S Chan, Alain Dufresne, and Michael K Danquah. "Review on Nanoparticles and Nanostructured Materials: History, Sources, Toxicity and Regulations.” Beilstein Journal of Nanotechnology 9 (April 3, 2018): 1050-1074. doi:10.3762/bjnano.9.98.

[12] Mostafavi, Ebrahim, Pooneh Soltantabar, and Thomas J. Webster. "Nanotechnology and Picotechnology." Biomaterials in Translational Medicine (2019): 191-212. doi:10.1016/b978-0-12-813477-1.00009-8.

[13] Nasrollahzadeh, Mahmoud, Mohaddeseh Sajjadi, S. Mohammad Sajadi, and Zahra Issaabadi. “Green Nanotechnology.” An Introduction to Green Nanotechnology (2019): 145-198. doi:10.1016/b978-0-12-813586-0.00005-5.

[14] Jeevanandam, Jaison, Yen San Chan, and Michael K. Danquah. "Biosynthesis of Metal and Metal Oxide Nanoparticles." ChemBioEng Reviews 3, no. 2 (March 18, 2016): 55-67. doi:10.1002/cben.201500018. 
[15] Fabiano, Bruno, Andrea P. Reverberi, and Petar Sabev Varbanov. "Safety Opportunities for the Synthesis of Metal Nanoparticles and Short-Cut Approach to Workplace Risk Evaluation.” Journal of Cleaner Production 209 (February 2019): $297-308$. doi:10.1016/j.jclepro.2018.10.161.

[16] Jeevanandam, Jaison, Yen San Chan, and Michael K. Danquah. "Nano-Formulations of Drugs: Recent Developments, Impact and Challenges.” Biochimie 128-129 (September 2016): 99-112. doi:10.1016/j.biochi.2016.07.008.

[17] Pal, Gaurav, Priya Rai, and Anjana Pandey. "Green Synthesis of Nanoparticles: A Greener Approach for a Cleaner Future." Green Synthesis, Characterization and Applications of Nanoparticles (2019): 1-26. doi:10.1016/b978-0-08-102579-6.00001-0.

[18] Yang, Cuiping, Tianhong Zhang, Zheng Li, Liang Xu, Fei Liu, Jinxiu Ruan, Keliang Liu, and Zhenqing Zhang. "P-Glycoprotein Is Responsible for the Poor Intestinal Absorption and Low Toxicity of Oral Aconitine: In Vitro, in Situ, in Vivo and in Silico Studies.” Toxicology and Applied Pharmacology 273, no. 3 (December 2013): 561-568. doi:10.1016/j.taap.2013.09.030.

[19] Jones, Clinton F., and David W. Grainger. "In Vitro Assessments of Nanomaterial Toxicity.” Advanced Drug Delivery Reviews 61, no. 6 (June 2009): 438-456. doi:10.1016/j.addr.2009.03.005.

[20] Cohen, Yoram, Robert Rallo, Rong Liu, and HAOYANG HAVEN Liu. "In Silico Analysis of Nanomaterials Hazard and Risk." Accounts of Chemical Research 46, no. 3 (November 8, 2012): 802-812. doi:10.1021/ar300049e.

[21] Katt, Moriah E., Amanda L. Placone, Andrew D. Wong, Zinnia S. Xu, and Peter C. Searson. "In Vitro Tumor Models: Advantages, Disadvantages, Variables, and Selecting the Right Platform." Frontiers in Bioengineering and Biotechnology 4 (February 12, 2016). doi:10.3389/fbioe.2016.00012.

[22] Godin, B, and E Touitou. "Transdermal Skin Delivery: Predictions for Humans from in Vivo, Ex Vivo and Animal Models." Advanced Drug Delivery Reviews 59, no. 11 (September 30, 2007): 1152 - 1161. doi:10.1016/j.addr.2007.07.004.

[23] Saeidnia, Soodabeh, Azadeh Manayi, and Mohammad Abdollahi. "From in Vitro Experiments to in Vivo and Clinical Studies; Pros and Cons." Current Drug Discovery Technologies 12, no. 4 (January 22, 2016): 218-224. doi:10.2174/1570163813666160114093140

[24] Cheng, Delfine, Gerald J. Shami, Marco Morsch, Roger S. Chung, and Filip Braet. "Ultrastructural Mapping of the Zebrafish Gastrointestinal System as a Basis for Experimental Drug Studies.” BioMed Research International 2016 (2016): 1-13. doi:10.1155/2016/8758460.

[25] Yang, Feng, Wenhui Qiu, Rongzhen Li, Jiaqi Hu, Shusheng Luo, Ting Zhang, Xin He, and Chunmiao Zheng. "Genome-Wide Identification of the Interactions Between Key Genes and Pathways Provide New Insights into the Toxicity of Bisphenol F and S During Early Development in Zebrafish.” Chemosphere 213 (December 2018): $559-567$. doi:10.1016/j.chemosphere.2018.09.133.

[26] MacRae, Calum A., and Randall T. Peterson. “Zebrafish as Tools for Drug Discovery.” Nature Reviews Drug Discovery 14, no. 10 (September 11, 2015): 721-731. doi:10.1038/nrd4627.

[27] Li, Zhen Hua, Deepa Alex, Shiu On Siu, Ivan Keung Chu, Joerg Renn, Christoph Winkler, Shaoke Lou, et al. "Combined in Vivo Imaging and Omics Approaches Reveal Metabolism of Icaritin and Its Glycosides in Zebrafish Larvae." Molecular BioSystems 7, no. 7 (2011): 2128. doi:10.1039/c1mb00001b.

[28] Parng, Chuenlei, Wen Lin Seng, Carlos Semino, and Patricia McGrath. "Zebrafish: A Preclinical Model for Drug Screening." ASSAY and Drug Development Technologies 1, no. 1 (November 2002): 41-48. doi:10.1089/154065802761001293.

[29] Wertman, Jaime, Chansey J. Veinotte, Graham Dellaire, and Jason N. Berman. "The Zebrafish Xenograft Platform: Evolution of a Novel Cancer Model and Preclinical Screening Tool.” Advances in Experimental Medicine and Biology (2016): $289-314$. doi:10.1007/978-3-319-30654-4_13.

[30] Lenard, A., E. Alghisi, H. Daff, M. Donzelli, C. McGinnis, and C. Lengerke. "Using Zebrafish to Model Erythroid Lineage Toxicity and Regeneration.” Haematologica 101, no. 5 (March 4, 2016): e164-e167. doi:10.3324/haematol.2016.142562.

[31] Dang, Michelle, Rachel E. Henderson, Levi A. Garraway, and Leonard I. Zon. "Long-Term Drug Administration in the Adult Zebrafish Using Oral Gavage for Cancer Preclinical Studies.” Disease Models \& Mechanisms 9, no. 7 (May 11, 2016): 811820. doi:10.1242/dmm.024166.

[32] Ludolph, Albert C., Caterina Bendotti, Eran Blaugrund, Adriano Chio, Linda Greensmith, Jean-Philippe Loeffler, Richard Mead, et al. "Guidelines for Preclinical Animal Research in ALS/MND: A Consensus Meeting." Amyotrophic Lateral Sclerosis 11, no. 1-2 (January 2010): 38-45. doi:10.3109/17482960903545334.

[33] Jucker, Mathias. "The Benefits and Limitations of Animal Models for Translational Research in Neurodegenerative Diseases." Nature Medicine 16, no. 11 (September 21, 2010): 1210-1214. doi:10.1038/nm.2224.

[34] Kramer, T., B. Schmidt, and F. Lo Monte. "Small-Molecule Inhibitors of GSK-3: Structural Insights and Their Application to Alzheimer's Disease Models." International Journal of Alzheimer's Disease 2012, no. (2012): 32. doi: 10.1155/2012/381029. 
[35] Gut, P., S. Reischauer, D. Y. R. Stainier, and R. Arnaout. "Little Fish, Big Data: Zebrafish as a Model for Cardiovascular and Metabolic Disease." Physiological Reviews 97, no. 3 (2017): 889-938. doi: 10.1152/physrev.00038.2016.

[36] Oksanen, Kaisa E., Nicholas J.A. Halfpenny, Eleanor Sherwood, Sanna-Kaisa E. Harjula, Milka M. Hammarén, Maarit J. Ahava, Elina T. Pajula, Marika J. Lahtinen, Mataleena Parikka, and Mika Rämet. “An Adult Zebrafish Model for Preclinical Tuberculosis Vaccine Development.” Vaccine 31, no. 45 (October 2013): 5202-5209. doi:10.1016/j.vaccine.2013.08.093.

[37] Hersch, S. M., and R. J. Ferrante. "Translating therapies for Huntington's disease from genetic animal models to clinical trials." NeuroRX 1, no. 3 (2004): 298-306. doi: 10.1602/neurorx.1.3.298.

[38] Parker, Elizabeth U., and Lisa Maves. "Identifying Therapies for Muscle Disease Using Zebrafish." Stem Cell Biology and Regenerative Medicine (December 19, 2015): 281-293. doi:10.1007/978-1-4939-3228-3_10.

[39] Torres, V. E. "Pro: Tolvaptan delays the progression of autosomal dominant polycystic kidney disease." Nephrology Dialysis Transplantation 34, no. 1 (2018): 30-34. doi: 10.1093/ndt/gfy297.

[40] Zhu, Q., X. Zhuang, and J. Lu. "Neuroprotective effects of baicalein in animal models of Parkinson's disease: A systematic review of experimental studies." Phytomedicine no. (2018). doi: 10.1016/j.phymed.2018.09.215.

[41] Martz, Harry E., Clint M. Logan, Daniel J. Schneberk, and Peter J. Shull. "X-Ray Imaging” (October 26, 2016). doi:10.1201/9781315375199.

[42] Bourassa, Daisy, Sophie-Charlotte Gleber, Stefan Vogt, Hong Yi, Fabian Will, Heiko Richter, Chong Hyun Shin, and Christoph J. Fahrni. "3D Imaging of Transition Metals in the Zebrafish Embryo by X-Ray Fluorescence Microtomography." Metallomics 6, no. 9 (July 4, 2014): 1648. doi:10.1039/c4mt00121d.

[43] Cheng, Keith C, Xuying Xin, Darin P Clark, and Patrick La Riviere. "Whole-Animal Imaging, Gene Function, and the Zebrafish Phenome Project." Current Opinion in Genetics \& Development 21, no. 5 (October 2011): 620-629. doi:10.1016/j.gde.2011.08.006.

[44] Seo, Eunseok, Jae-Hong Lim, Seung Jun Seo, and Sang Joon Lee. "Whole-Body Imaging of a Hypercholesterolemic Female Zebrafish by Using Synchrotron X-Ray Micro-CT.” Zebrafish 12, no. 1 (February 2015): 11-20. doi:10.1089/zeb.2014.1039.

[45] Vågberg, William, Daniel H. Larsson, Mei Li, Anders Arner, and Hans M. Hertz. "X-Ray Phase-Contrast Tomography for HighSpatial-Resolution Zebrafish Muscle Imaging.” Scientific Reports 5, no. 1 (November 13, 2015). doi:10.1038/srep16625.

[46] Plouraboue, F., P. Cloetens, C. Fonta, A. Steyer, F. Lauwers, and J.-P. Marc-Vergnes. "X-Ray High-Resolution Vascular Network Imaging." Journal of Microscopy 215, no. 2 (August 2004): 139-148. doi:10.1111/j.0022-2720.2004.01362.x.

[47] Pushie, M. Jake, Ingrid J. Pickering, Malgorzata Korbas, Mark J. Hackett, and Graham N. George. "Elemental and Chemically Specific X-Ray Fluorescence Imaging of Biological Systems." Chemical Reviews 114, no. 17 (August 7, 2014): 8499-8541. doi:10.1021/cr4007297.

[48] Ferstl, Simone, Brian Metscher, Mark Müller, Sebastian Allner, Martin Dierolf, Madleen Busse, Klaus Achterhold, Bernhard Gleich, and Franz Pfeiffer. "Laboratory-Based X-Ray NanoCT Explores Morphology of a Zebrafish Embryo.” Microscopy and Microanalysis 24, no. S2 (August 2018): 184-185. doi:10.1017/s1431927618013272.

[49] Xu, Quan, Miaoran Zhang, Yao Liu, Wei Cai, Wenjing Yang, Ziying He, Xiuli Sun, Yan Luo, and Fang Liu. "Synthesis of Multi-Functional Green Fluorescence Carbon Dots and Their Applications as a Fluorescent Probe for Hg2+ Detection and Zebrafish Imaging.” New Journal of Chemistry 42, no. 12 (2018): 10400-10405. doi:10.1039/c8nj01639a.

[50] Hertz, Hans. "Liquid-metal-jet x-ray sources and high-resolution biomedical imaging." In APS Meeting Abstracts. 2018.

[51] Yaniv, Karina, Sumio Isogai, Daniel Castranova, Louis Dye, Jiro Hitomi, and Brant M Weinstein. "Live Imaging of Lymphatic Development in the Zebrafish.” Nature Medicine 12, no. 6 (May 28, 2006): 711-716. doi:10.1038/nm1427.

[52] Sun, Lei, Ching-Ling Lien, Xiaochen Xu, and K. Kirk Shung. "In Vivo Cardiac Imaging of Adult Zebrafish Using High Frequency Ultrasound (45-75 MHz)." Ultrasound in Medicine \& Biology 34, no. 1 (January 2008): 31-39. doi:10.1016/j.ultrasmedbio.2007.07.002.

[53] Bassi, Andrea, Luca Fieramonti, Cosimo D’Andrea, Marina Mione, and Gianluca Valentini. "In Vivo Label-Free ThreeDimensional Imaging of Zebrafish Vasculature with Optical Projection Tomography.” Journal of Biomedical Optics 16, no. 10 (2011): 100502. doi:10.1117/1.3640808.

[54] Ma, Rui, Martin Distel, X Luís Deán-Ben, Vasilis Ntziachristos, and Daniel Razansky. "Non-Invasive Whole-Body Imaging of Adult Zebrafish with Optoacoustic Tomography.” Physics in Medicine and Biology 57, no. 22 (October 17, 2012): $7227-7237$. doi:10.1088/0031-9155/57/22/7227.

[55] Correia, Teresa, Nicola Lockwood, Sunil Kumar, Jun Yin, Marie-Christine Ramel, Natalie Andrews, Matilda Katan, et al. “Accelerated Optical Projection Tomography Applied to In Vivo Imaging of Zebrafish.” Edited by Thomas Abraham. PLOS ONE 10, no. 8 (August 26, 2015): e0136213. doi:10.1371/journal.pone.0136213. 
[56] Vedula, Vijay, Juhyun Lee, Hao Xu, C.-C. Jay Kuo, Tzung K. Hsiai, and Alison L. Marsden. "A Method to Quantify Mechanobiologic Forces During Zebrafish Cardiac Development Using 4-D Light Sheet Imaging and Computational Modeling." Edited by Scott L Diamond. PLOS Computational Biology 13, no. 10 (October 30, 2017): e1005828. doi:10.1371/journal.pcbi.1005828.

[57] Deán-Ben, X. Luís, Sven Gottschalk, Gali Sela, Shy Shoham, and Daniel Razansky. "Functional Optoacoustic NeuroTomography of Calcium Fluxes in Adult Zebrafish Brain in Vivo." Optics Letters 42, no. 5 (February 27, 2017 ): 959. doi:10.1364/ol.42.000959.

[58] Kang, Yan-Fei, Yu-Hao Li, Yang-Wu Fang, Yang Xu, Xiao-Mi Wei, and Xue-Bo Yin. "Carbon Quantum Dots for Zebrafish Fluorescence Imaging." Scientific Reports 5, no. 1 (July 2, 2015). doi:10.1038/srep11835.

[59] Parng, Chuenlei, Nicole Marie Roy, Christopher Ton, Yingxin Lin, and Patricia McGrath. "Neurotoxicity Assessment Using Zebrafish." Journal of Pharmacological and Toxicological Methods 55, no. 1 (January 2007): $103-112$. doi:10.1016/j.vascn.2006.04.004.

[60] Kokel, David, Jennifer Bryan, Christian Laggner, Rick White, Chung Yan J Cheung, Rita Mateus, David Healey, et al. "Rapid Behavior-Based Identification of Neuroactive Small Molecules in the Zebrafish.” Nature Chemical Biology 6, no. 3 (January 17, 2010): 231-237. doi:10.1038/nchembio.307.

[61] Panula, P., Y.-C. Chen, M. Priyadarshini, H. Kudo, S. Semenova, M. Sundvik, and V. Sallinen. "The Comparative Neuroanatomy and Neurochemistry of Zebrafish CNS Systems of Relevance to Human Neuropsychiatric Diseases." Neurobiology of Disease 40, no. 1 (October 2010): 46-57. doi:10.1016/j.nbd.2010.05.010.

[62] Groth, Casper, Svanhild Nornes, Rosa McCarty, Richard Tamme, and Michael Lardelli. "Identification of a second presenilin gene in zebrafish with similarity to the human Alzheimer's disease gene presenilin2." Development genes and evolution 212, no. 10 (2002): 486-490. doi: 10.1007/s00427-002-0269-5.

[63] Newman, Morgan, Giuseppe Verdile, Ralph N. Martins, and Michael Lardelli. "Zebrafish as a tool in Alzheimer's disease research." Biochimica et Biophysica Acta (BBA)-Molecular Basis of Disease 1812, no. 3 (2011): 346-352. doi:10.1016/j.bbadis.2010.09.012.

[64] Richetti, S.K., M. Blank, K.M. Capiotti, A.L. Piato, M.R. Bogo, M.R. Vianna, and C.D. Bonan. "Quercetin and Rutin Prevent Scopolamine-Induced Memory Impairment in Zebrafish.” Behavioural Brain Research 217, no. 1 (February 2011): 10-15. doi:10.1016/j.bbr.2010.09.027.

[65] Geling, A. "A Gamma-Secretase Inhibitor Blocks Notch Signaling in Vivo and Causes a Severe Neurogenic Phenotype in Zebrafish.” EMBO Reports 3, no. 7 (July 15, 2002): 688-694. doi:10.1093/embo-reports/kvf124.

[66] Panula, Pertti, Ville Sallinen, Maria Sundvik, Juha Kolehmainen, Veera Torkko, Anu Tiittula, Maxim Moshnyakov, and Piotr Podlasz. "Modulatory Neurotransmitter Systems and Behavior: Towards Zebrafish Models of Neurodegenerative Diseases." Zebrafish 3, no. 2 (June 2006): 235-247. doi:10.1089/zeb.2006.3.235.

[67] Baulac, Stéphanie, Hope Lu, Jennifer Strahle, Ting Yang, Matthew S. Goldberg, Jie Shen, Michael G. Schlossmacher, Cynthia A. Lemere, Qun Lu, and Weiming Xia. "Increased DJ-1 expression under oxidative stress and in Alzheimer's disease brains." Molecular neurodegeneration 4, no. 1 (2009): 12. doi: 10.1186/1750-1326-4-12.

[68] Koehler, Daniel, Zahoor A. Shah, Kenneth Hensley, and Frederick E. Williams. "Lanthionine ketimine-5-ethyl ester provides neuroprotection in a zebrafish model of okadaic acid-induced Alzheimer's disease." Neurochemistry international 115 (2018): 61-68. doi: 10.1016/j.neuint.2018.02.002.

[69] Hin, N., M. Newman, J. Kaslin, A. M. Douek, A. Lumsden, X.-F. Zhou, N. B. Mañucat-Tan, A. Ludington, D. L. Adelson, and S. Pederson. "Accelerated brain aging towards transcriptional inversion in a zebrafish model of familial Alzheimer's disease." BioRxiv no. (2018): 262162. doi: 10.1101/262162.

[70] Koehler, Daniel, Zahoor A. Shah, and Frederick E. Williams. "The GSK3 $\beta$ inhibitor, TDZD-8, rescues cognition in a zebrafish model of okadaic acid-induced Alzheimer's disease." Neurochemistry international 122 (2019): 31-37. doi: 10.1016/j.neuint.2018.10.022.

[71] Mani, R. J., K. Mittal, and D. P. Katare. "Protective Effects of Quercetin in Zebrafish Model of Alzheimer's Disease." Asian Journal of Pharmaceutics 12, no. 2 (June 1, 2018): S660-S666. doi:10.22377/ajp.v12i02.2412.

[72] Lee, Jinyoung, Samuel M. Peterson, and Jennifer L. Freeman. “Alzheimer's Disease Risk Genes in Wild-Type Adult Zebrafish Exhibit Gender-Specific Expression Changes During Aging.” Neurogenetics 17, no. 3 (May 28, 2016): 197-199. doi:10.1007/s10048-016-0485-1.

[73] Wang, Yali, Wenwen Liu, Jing Yang, Fen Wang, Yizhen Sima, Zhao-min Zhong, Han Wang, Li-Fang Hu, and Chun-Feng Liu. “Parkinson's Disease-Like Motor and Non-Motor Symptoms in Rotenone-Treated Zebrafish." NeuroToxicology 58 (January 2017): 103-109. doi:10.1016/j.neuro.2016.11.006. 
[74] Sarath Babu, Nukala, Ch Lakshmi N. Murthy, Sameera Kakara, Rahul Sharma, Cherukuvada V. Brahmendra Swamy, and Mohammed M. Idris. "1 - Methyl - 4 - phenyl - 1, 2, 3, 6 - tetrahydropyridine induced Parkinson's disease in zebrafish." Proteomics 16, no. 9 (2016): 1407-1420. doi: 10.1002/pmic.201500291.

[75] Veldman, Matthew B., Yesenia Rios-Galdamez, Xiao-Hong Lu, Xiaofeng Gu, Wei Qin, Song Li, X. William Yang, and Shuo Lin. "The N17 Domain Mitigates Nuclear Toxicity in a Novel Zebrafish Huntington's Disease Model." Molecular Neurodegeneration 10, no. 1 (December 2015). doi:10.1186/s13024-015-0063-2.

[76] Chongtham, Anjalika, Brett Barbaro, Tomas Filip, Adeela Syed, Weijian Huang, Marianne R. Smith, and J. Lawrence Marsh. “Nonmammalian Models of Huntington's Disease.” Huntington's Disease (2018): 75-96. doi:10.1007/978-1-4939-7825-0_5.

[77] Rounding, Natalie. "Zebrafish C9orf72 loss-of-function models of Amyotrophic Lateral Sclerosis and Frontotemporal Dementia." PhD diss., University of Sheffield, 2018.

[78] Lin, C.-Y., P.-H. Zhang, Y.-J. Chen, C.-L. Wu, and H.-J. Tsai. "Conditional Overexpression of rtn4al in Muscle of Adult Zebrafish Displays Defects Similar to Human Amyotrophic Lateral Sclerosis." Marine Biotechnology 21, no. 1 (2019): 52-64. doi: $10.1007 / \mathrm{s} 10126-018-9857-\mathrm{x}$.

[79] Mayrhofer, M., V. Gourain, M. Reischl, P. Affaticati, A. Jenett, J.-S. Joly, M. Benelli, F. Demichelis, P. L. Poliani, D. Sieger, and M. Mione. "A novel brain tumour model in zebrafish reveals the role of YAP activation in MAPK- and PI3K-induced malignant growth." Disease Models \&amp;amp; Mechanisms 10, no. 1 (2017): 15. doi: 10.1242/dmm.026500.

[80] Li, S., Z. Peng, J. Dallman, J. Baker, A. M. Othman, P. L. Blackwelder, and R. M. Leblanc. "Crossing the blood-brain-barrier with transferrin conjugated carbon dots: A zebrafish model study." Colloids and Surfaces B: Biointerfaces 145, no. (2016): 251256. doi: 10.1016/j.colsurfb.2016.05.007.

[81] Lee, I. J., Y.-Q. Yang, W.-T. Chang, and I. Liau. "Abstract 123: Establishing a Zebrafish Model of Ischemic Stroke Induced by Photochemical Thrombosis: a Novel Platform for Translational Researches." Arteriosclerosis, Thrombosis, and Vascular Biology 38, no. Suppl_1 (2018): A123-A123. doi: 10.1161/atvb.38.suppl_1.123.

[82] Lee, I-Ju, Jia-Wen Hsu, Yung-Jen Chuang, and Ian Liau. "Confocal Imaging Guided Photochemical Thrombosis Toward the Development of a Novel Zebrafish Model of Stroke.” Asia Communications and Photonics Conference 2016 (2016). doi:10.1364/acpc.2016.as3i.3.

[83] Scholz, S., S. Fischer, U. Gündel, E. Küster, T. Luckenbach, and D. Voelker. "The zebrafish embryo model in environmental risk assessment — applications beyond acute toxicity testing." Environmental Science and Pollution Research 15, no. 5 (2008): 394-404. doi: 10.1007/s11356-008-0018-z.

[84] Lammer, E., G.J. Carr, K. Wendler, J.M. Rawlings, S.E. Belanger, and Th. Braunbeck. "Is the Fish Embryo Toxicity Test (FET) with the Zebrafish (Danio Rerio) a Potential Alternative for the Fish Acute Toxicity Test?" Comparative Biochemistry and Physiology Part C: Toxicology \& Pharmacology 149, no. 2 (March 2009): 196-209. doi:10.1016/j.cbpc.2008.11.006.

[85] Hill, Adrian J., Hiroki Teraoka, Warren Heideman, and Richard E. Peterson. "Zebrafish as a Model Vertebrate for Investigating Chemical Toxicity.” Toxicological Sciences 86, no. 1 (February 9, 2005): 6-19. doi:10.1093/toxsci/kfi110.

[86] Carney, S. A., A. L. Prasch, W. Heideman, and R. E. Peterson. "Understanding dioxin developmental toxicity using the zebrafish model." Birth Defects Research Part A: Clinical and Molecular Teratology 76, no. 1 (2006): 7-18. doi: 10.1002/bdra.20216.

[87] Hagenaars, A., L. Vergauwen, W. De Coen, and D. Knapen. "Structure-activity relationship assessment of four perfluorinated chemicals using a prolonged zebrafish early life stage test." Chemosphere 82, no. 5 (2011): 764-772. doi:10.1016/j.chemosphere.2010.10.076.

[88] Hallare, A., K. Nagel, H.-R. Köhler, and R. Triebskorn. "Comparative embryotoxicity and proteotoxicity of three carrier solvents to zebrafish (Danio rerio) embryos." Ecotoxicology and Environmental Safety 63, no. 3 (2006): 378-388. doi:10.1016/j.ecoenv.2005.07.006.

[89] Dave, G., and R. Xiu. "Toxicity of mercury, copper, nickel, lead, and cobalt to embryos and larvae of zebrafish,Brachydanio rerio." Archives of Environmental Contamination and Toxicology 21, no. 1 (1991): 126-134. doi: 10.1007/BF01055567.

[90] Babayigit, Aslihan, Dinh Duy Thanh, Anitha Ethirajan, Jean Manca, Marc Muller, Hans-Gerd Boyen, and Bert Conings. “Assessing the Toxicity of Pb- and Sn-Based Perovskite Solar Cells in Model Organism Danio Rerio.” Scientific Reports 6, no. 1 (January 13, 2016). doi:10.1038/srep18721.

[91] Kienle, Cornelia, Heinz-R. Köhler, and Almut Gerhardt. "Behavioural and Developmental Toxicity of Chlorpyrifos and Nickel Chloride to Zebrafish (Danio Rerio) Embryos and Larvae.” Ecotoxicology and Environmental Safety 72, no. 6 (September 2009): 1740-1747. doi:10.1016/j.ecoenv.2009.04.014.

[92] Cheng, S. H., A. W. K. Wai, C. H. So, and R. S. S. Wu. "Cellular and molecular basis of cadmium-induced deformities in zebrafish embryos." Environmental Toxicology and Chemistry 19, no. 12 (2000): 3024-3031. doi: 10.1002/etc.5620191223. 
[93] Yin, J., A.-P. Wang, W.-F. Li, R. Shi, H.-T. Jin, and J.-F. Wei. "Time-response characteristic and potential biomarker identification of heavy metal induced toxicity in zebrafish." Fish \& Shellfish Immunology 72, no. (2018): 309-317. doi:10.1016/j.fsi.2017.10.047.

[94] Lu, Kai, Ruxia Qiao, Hao An, and Yan Zhang. "Influence of Microplastics on the Accumulation and Chronic Toxic Effects of Cadmium in Zebrafish (Danio Rerio).” Chemosphere 202 (July 2018): 514-520. doi:10.1016/j.chemosphere.2018.03.145.

[95] Liu, T., R. Wang, H. Cao, and A. Lin. "Polyaspartic acid alleviates heavy metal toxicity in zebrafish (Danio rerio)." Chemistry and Ecology 33, no. 7 (2017): 684-693. doi: 10.1080/02757540.2017.1351959.

[96] Alkilany, Alaaldin M., and Catherine J. Murphy. "Toxicity and Cellular Uptake of Gold Nanoparticles: What We Have Learned so Far?” Journal of Nanoparticle Research 12, no. 7 (April 6, 2010): 2313-2333. doi:10.1007/s11051-010-9911-8.

[97] Baek, Yong-Wook, and Youn-Joo An. "Microbial Toxicity of Metal Oxide Nanoparticles (CuO, NiO, ZnO, and Sb2O3) to Escherichia Coli, Bacillus Subtilis, and Streptococcus Aureus." Science of The Total Environment 409, no. 8 (March 2011): 1603-1608. doi:10.1016/j.scitotenv.2011.01.014.

[98] Lee, Jungwoo, G. Daniel Lilly, R. Christopher Doty, Paul Podsiadlo, and Nicholas A. Kotov. "In Vitro Toxicity Testing of Nanoparticles in 3D Cell Culture.” Small (March 4, 2009): 1213-1221. doi:10.1002/smll.200801788.

[99] Bahadar, Haji, Faheem Maqbool, Kamal Niaz, and Mohammad Abdollahi. "Toxicity of nanoparticles and an overview of current experimental models." Iranian biomedical journal 20, no. 1 (2016): 1. doi: 10.7508/ibj.2016.01.001.

[100] Small, Taika, M. Amparo Ochoa-Zapater, Gianni Gallello, Antonio Ribera, Francisco M. Romero, Amparo Torreblanca, and M. Dolores Garcerá. "Gold-Nanoparticles Ingestion Disrupts Reproduction and Development in the German Cockroach." Science of The Total Environment 565 (September 2016): 882-888. doi:10.1016/j.scitotenv.2016.02.032.

[101] Alaraby, Mohamed, Balasubramanyam Annangi, Ricard Marcos, and Alba Hernández. "Drosophila Melanogasteras a Suitable in Vivo Model to Determine Potential Side Effects of Nanomaterials: A Review.” Journal of Toxicology and Environmental Health, Part B 19, no. 2 (February 17, 2016): 65-104. doi:10.1080/10937404.2016.1166466.

[102] Jozefczuk, Justyna, Alessandro Prigione, Lukas Chavez, and James Adjaye. "Comparative Analysis of Human Embryonic Stem Cell and Induced Pluripotent Stem Cell-Derived Hepatocyte-Like Cells Reveals Current Drawbacks and Possible Strategies for Improved Differentiation.” Stem Cells and Development 20, no. 7 (July 2011): 1259-1275. doi:10.1089/scd.2010.0361.

[103] Ukelis, Ute, Peter-Jürgen Kramer, Klaus Olejniczak, and Stefan O. Mueller. "Replacement of in Vivo Acute Oral Toxicity Studies by in Vitro Cytotoxicity Methods: Opportunities, Limits and Regulatory Status." Regulatory Toxicology and Pharmacology 51, no. 1 (June 2008): 108-118. doi:10.1016/j.yrtph.2008.02.002.

[104] Lin, Sijie, Yan Zhao, André E. Nel, and Shuo Lin. "Zebrafish: An In Vivo Model for Nano EHS Studies." Small 9, no. 9-10 (December 3, 2012): 1608-1618. doi:10.1002/smll.201202115.

[105] Mizgirev, Igor, and Sergei Revskoy. "Generation of Clonal Zebrafish Lines and Transplantable Hepatic Tumors." Nature Protocols 5, no. 3 (February 11, 2010): 383-394. doi:10.1038/nprot.2010.8.

[106] Haque, Enamul, and Alister Ward. "Zebrafish as a Model to Evaluate Nanoparticle Toxicity.” Nanomaterials 8, no. 7 (July 23, 2018): 561. doi:10.3390/nano8070561.

[107] Wehmas, Leah C., Catherine Anders, Jordan Chess, Alex Punnoose, Cliff B. Pereira, Juliet A. Greenwood, and Robert L. Tanguay. "Comparative Metal Oxide Nanoparticle Toxicity Using Embryonic Zebrafish.” Toxicology Reports 2 (2015): 702715. doi:10.1016/j.toxrep.2015.03.015.

[108] Della Torre, Camilla, Marco Parolini, Luca Del Giacco, Anna Ghilardi, Miriam Ascagni, Nadia Santo, Daniela Maggioni, et al. "Adsorption of $\mathrm{B}(\alpha) \mathrm{P}$ on Carbon Nanopowder Affects Accumulation and Toxicity in Zebrafish (Danio Rerio) Embryos." Environmental Science: Nano 4, no. 5 (2017): 1132-1146. doi:10.1039/c7en00154a.

[109] Hu, Qinglian, Fengliang Guo, Fenghui Zhao, Guping Tang, and Zhengwei Fu. "Cardiovascular Toxicity Assessment of Poly (ethylene Imine)- Based Cationic Polymers on Zebrafish Model.” Journal of Biomaterials Science, Polymer Edition 28, no. 8 (March 9, 2017): 768-780. doi:10.1080/09205063.2017.1301773.

[110] Pecoraro, Roberta, Antonio Salvaggio, Fabio Marino, Gianfranco Di Caro, Fabiano Capparucci, Bianca Maria Lombardo, Giuseppina Messina, et al. "Metallic Nano-Composite Toxicity Evaluation by Zebrafish Embryo Toxicity Test with Identification of Specific Exposure Biomarkers.” Current Protocols in Toxicology (November 8, 2017): 1.14.1-1.14.13. doi:10.1002/cptx.34.

[111] Wang, Ruhung, Alicea N. Meredith, Michael Lee, Dakota Deutsch, Lizaveta Miadzvedskaya, Elizabeth Braun, Paul Pantano, Stacey Harper, and Rockford Draper. "Toxicity Assessment and Bioaccumulation in Zebrafish Embryos Exposed to Carbon Nanotubes Suspended in Pluronic ${ }^{\circledR}$ F-108.” Nanotoxicology 10, no. 6 (November 11, 2015): 689-698. doi:10.3109/17435390.2015.1107147. 
[112] Manjunatha, Bangeppagari, Sung Ho Park, Kiwoong Kim, Rajesh R Kundapur, and Sang Joon Lee. "In Vivo Toxicity Evaluation of Pristine Graphene in Developing Zebrafish (Danio Rerio) Embryos.” Environmental Science and Pollution Research 25, no. 13 (February 23, 2018): 12821-12829. doi:10.1007/s11356-018-1420-9.

[113] Sandhöfer, Benedikt, Marian Meckel, José Manuel Delgado-López, Tatiana Patrício, Anna Tampieri, Frank Rösch, and Michele Iafisco. "Synthesis and Preliminary in Vivo Evaluation of Well-Dispersed Biomimetic Nanocrystalline Apatites Labeled with Positron Emission Tomographic Imaging Agents.” ACS Applied Materials \& Interfaces 7, no. 19 (May 7, 2015): $10623-10633$. doi:10.1021/acsami.5b02624.

[114] Lemos, J., T. Neuparth, M. Trigo, P. Costa, D. Vieira, L. Cunha, F. Ponte, P. S. Costa, L. F. Metello, and A. P. Carvalho. "Single Low-Dose Ionizing Radiation Induces Genotoxicity in Adult Zebrafish and Its Non-Irradiated Progeny." Bulletin of Environmental Contamination and Toxicology 98, no. 2 (December 26, 2016): 190-195. doi:10.1007/s00128-016-2006-1.

[115] Ramachandran, R., C. Krishnaraj, V. K. A. Kumar, S. L. Harper, T. P. Kalaichelvan, and S.-I. Yun. "In vivo toxicity evaluation of biologically synthesized silver nanoparticles and gold nanoparticles on adult zebrafish: a comparative study." 3 Biotech 8 , no. 10 (2018): 441. doi: 10.1007/s13205-018-1457-y.

[116] Ramachandran, R., C. Krishnaraj, A. S. Sivakumar, P. Prasannakumar, V. K. Abhay Kumar, K. S. Shim, C.-G. Song, and S.-I. Yun. "Anticancer activity of biologically synthesized silver and gold nanoparticles on mouse myoblast cancer cells and their toxicity against embryonic zebrafish." Materials Science and Engineering: C 73, no. (2017): 674-683. doi:10.1016/j.msec.2016.12.110.

[117] Groh, K. J., T. Dalkvist, F. Piccapietra, R. Behra, M. J. F. Suter, and K. Schirmer. "Critical influence of chloride ions on silver ion-mediated acute toxicity of silver nanoparticles to zebrafish embryos." Nanotoxicology 9, no. 1 (2015): 81-91. doi:10.3109/17435390.2014.893379.

[118] Pecoraro, R., F. Marino, A. Salvaggio, F. Capparucci, G. Di Caro, C. Iaria, A. Salvo, A. Rotondo, D. Tibullo, G. Guerriero, E. M. Scalisi, M. Zimbone, G. Impellizzeri, and M. V. Brundo. "Evaluation of Chronic Nanosilver Toxicity to Adult Zebrafish." Frontiers in Physiology 8, no. 1011 (2017). doi:10.3389/fphys.2017.01011.

[119] Denluck, L., F. Wu, L. E. Crandon, B. J. Harper, and S. L. Harper. "Reactive oxygen species generation is likely a driver of copper based nanomaterial toxicity." Environmental Science: Nano 5, no. 6 (2018): 1473-1481. doi: 10.1039/C8EN00055G.

[120] Kalishwaralal, K., S. Jeyabharathi, K. Sundar, and A. Muthukumaran. "A novel one-pot green synthesis of selenium nanoparticles and evaluation of its toxicity in zebrafish embryos." Artificial Cells, Nanomedicine, and Biotechnology 44, no. 2 (2016): 471-477. doi: 10.3109/21691401.2014.962744.

[121] Asharani, P. V., Y. lianwu, Z. Gong, and S. Valiyaveettil. "Comparison of the toxicity of silver, gold and platinum nanoparticles in developing zebrafish embryos." Nanotoxicology 5, no. 1 (2011): 43-54. doi: 10.3109/17435390.2010.489207.

[122] Griffitt, R. J., A. Feswick, R. Weil, K. Hyndman, P. Carpinone, K. Powers, N. D. Denslow, and D. S. Barber. "Investigation of acute nanoparticulate aluminum toxicity in zebrafish." Environmental Toxicology 26, no. 5 (2011): 541-551. doi:10.1002/tox.20669.

[123] Kteeba, S. M., H. I. El-Adawi, O. A. El-Rayis, A. E. El-Ghobashy, J. L. Schuld, K. R. Svoboda, and L. Guo. "Zinc oxide nanoparticle toxicity in embryonic zebrafish: Mitigation with different natural organic matter." Environmental Pollution 230, no. (2017): 1125-1140. doi: 10.1016/j.envpol.2017.07.042.

[124] Thit, A., L. M. Skjolding, H. Selck, and J. Sturve. "Effects of copper oxide nanoparticles and copper ions to zebrafish (Danio rerio) cells, embryos and fry." Toxicology in Vitro 45, no. (2017): 89-100. doi: 10.1016/j.tiv.2017.08.010.

[125] Srinivasan, M., M. Venkatesan, V. Arumugam, G. Natesan, N. Saravanan, S. Murugesan, S. Ramachandran, R. Ayyasamy, and A. Pugazhendhi. "Green synthesis and characterization of titanium dioxide nanoparticles (TiO2 NPs) using Sesbania grandiflora and evaluation of toxicity in zebrafish embryos." Process Biochemistry 80, no. (2019): 197-202. doi:10.1016/j.procbio.2019.02.010.

[126] Jemec, A., P. Djinović, I. G. O. Črnivec, and A. Pintar. "The hazard assessment of nanostructured CeO2-based mixed oxides on the zebrafish Danio rerio under environmentally relevant UV-A exposure." Science of The Total Environment 506-507, no. (2015): 272-278. doi: 10.1016/j.scitotenv.2014.10.120.

[127] Ghobadian, M., M. Nabiuni, K. Parivar, M. Fathi, and J. Pazooki. "Toxic effects of magnesium oxide nanoparticles on early developmental and larval stages of zebrafish (Danio rerio)." Ecotoxicology and Environmental Safety 122, no. (2015): 260267. doi: 10.1016/j.ecoenv.2015.08.009.

[128] Vranic, S., Y. Shimada, S. Ichihara, M. Kimata, W. Wu, T. Tanaka, S. Boland, L. Tran, and G. Ichihara. "Toxicological Evaluation of SiO2 Nanoparticles by Zebrafish Embryo Toxicity Test." International Journal of Molecular Sciences 20, no. 4 (2019). doi: 10.3390/ijms20040882. 
[129] Villacis, R. A. R., J. S. Filho, B. Piña, R. B. Azevedo, A. Pic-Taylor, J. F. Mazzeu, and C. K. Grisolia. "Integrated assessment of toxic effects of maghemite $(\gamma$-Fe2O3) nanoparticles in zebrafish." Aquatic Toxicology 191, no. (2017): 219-225. doi:10.1016/j.aquatox.2017.08.004.

[130] Gilbertson, L. M., F. Melnikov, L. C. Wehmas, P. T. Anastas, R. L. Tanguay, and J. B. Zimmerman. "Toward safer multiwalled carbon nanotube design: Establishing a statistical model that relates surface charge and embryonic zebrafish mortality." Nanotoxicology 10, no. 1 (2016): 10-19. doi: 10.3109/17435390.2014.996193.

[131] Girardi, F. A., G. E. Bruch, C. S. Peixoto, L. Dal Bosco, S. K. Sahoo, C. O. F. Gonçalves, A. P. Santos, C. A. Furtado, C. Fantini, and D. M. Barros. "Toxicity of single-wall carbon nanotubes functionalized with polyethylene glycol in zebrafish (Danio rerio) embryos." Journal of Applied Toxicology 37, no. 2 (2017): 214-221. doi: 10.1002/jat.3346.

[132] Wang, Z. G., R. Zhou, D. Jiang, J. E. Song, Q. Xu, J. Si, Y. P. Chen, X. Zhou, L. Gan, J. Z. Li, H. Zhang, and B. Liu. "Toxicity of Graphene Quantum Dots in Zebrafish Embryo." Biomedical and Environmental Sciences 28, no. 5 (2015): $341-351$. doi:10.3967/bes2015.048.

[133] Dias, Cindy, Marisa P. Sárria, Nagamalai Vasimalai, Vânia Vilas-Boas, J. M. Peixoto, and Begoña Espiña. "Fruit-based carbon dots as fluorescent probes: in vitro and in vivo toxicity evaluation." (2017).

[134] Manjunatha, B., S. H. Park, K. Kim, R. R. Kundapur, and S. J. Lee. "In vivo toxicity evaluation of pristine graphene in developing zebrafish (Danio rerio) embryos." Environmental Science and Pollution Research 25, no. 13 (2018): 12821-12829. doi: 10.1007/s11356-018-1420-9.

[135] Soares, J. C., T. C. B. Pereira, K. M. Costa, T. Maraschin, N. R. Basso, and M. R. Bogo. "Developmental neurotoxic effects of graphene oxide exposure in zebrafish larvae (Danio rerio)." Colloids and Surfaces B: Biointerfaces 157, no. (2017): 335-346. doi: 10.1016/j.colsurfb.2017.05.078.

[136] Della Torre, C., D. Maggioni, A. Ghilardi, M. Parolini, N. Santo, C. Landi, L. Madaschi, S. Magni, S. Tasselli, M. Ascagni, L. Bini, C. La Porta, L. Del Giacco, and A. Binelli. "The interactions of fullerene C60 and Benzo $(\alpha)$ pyrene influence their bioavailability and toxicity to zebrafish embryos." Environmental Pollution 241, no. (2018): 999-1008. doi:10.1016/j.envpol.2018.06.042.

[137] Sarasamma, S., G. Audira, S. Juniardi, P. B. Sampurna, Y.-H. Lai, E. Hao, J.-R. Chen, and C.-D. Hsiao. "Evaluation of the Effects of Carbon 60 Nanoparticle Exposure to Adult Zebrafish: A Behavioral and Biochemical Approach to Elucidate the Mechanism of Toxicity." International Journal of Molecular Sciences 19, no. 12 (2018). doi: 10.3390/ijms19123853.

[138] Wang, Y., J. Zhou, L. Liu, C. Huang, D. Zhou, and L. Fu. "Characterization and toxicology evaluation of chitosan nanoparticles on the embryonic development of zebrafish, Danio rerio." Carbohydrate Polymers 141, no. (2016): 204-210. doi:10.1016/j.carbpol.2016.01.012.

[139] Zhao, X., K. J. Ong, J. D. Ede, J. L. Stafford, K. W. Ng, G. G. Goss, and S. C. J. Loo. "Evaluating the Toxicity of Hydroxyapatite Nanoparticles in Catfish Cells and Zebrafish Embryos." Small 9, no. 9 - 10 (2013): 1734-1741. doi: 10.1002/smll.201200639.

[140] Vibe, C. B., F. Fenaroli, D. Pires, S. R. Wilson, V. Bogoeva, R. Kalluru, M. Speth, E. Anes, G. Griffiths, and J. Hildahl. "Thioridazine in PLGA nanoparticles reduces toxicity and improves rifampicin therapy against mycobacterial infection in zebrafish." Nanotoxicology 10, no. 6 (2016): 680-688. doi: 10.3109/17435390.2015.1107146.

[141] Bodewein, L., F. Schmelter, S. Di Fiore, H. Hollert, R. Fischer, and M. Fenske. "Differences in toxicity of anionic and cationic PAMAM and PPI dendrimers in zebrafish embryos and cancer cell lines." Toxicology and Applied Pharmacology 305, no. (2016): 83-92. doi: 10.1016/j.taap.2016.06.008.

[142] He, N., X. Li, D. Feng, M. Wu, R. Chen, T. Chen, D. Chen, and X. Feng. "Exploring the Toxicity of a Bismuth-Asparagine Coordination Polymer on the Early Development of Zebrafish Embryos." Chemical Research in Toxicology 26, no. 1 (2013): 89-95. doi: 10.1021/tx3004032.

[143] Vong, L. B., M. Kobayashi, and Y. Nagasaki. "Evaluation of the Toxicity and Antioxidant Activity of Redox Nanoparticles in Zebrafish (Danio rerio) Embryos." Molecular Pharmaceutics 13, no. 9 (2016): 3091-3097. doi:10.1021/acs.molpharmaceut.6b00225.

[144] Nasrallah, G. K., R. Salem, S. Da'as, O. L. A. Al-Jamal, M. Scott, and I. Mustafa. "Biocompatibility and toxicity of novel iron chelator Starch-Deferoxamine (S-DFO) compared to zinc oxide nanoparticles to zebrafish embryo: An oxidative stress based apoptosis, physicochemical and neurological study profile." Neurotoxicology and Teratology 72, no. (2019): 29-38. doi:10.1016/j.ntt.2019.01.004.

[145] Prakash, J., M. Venkatesan, J. Sebastian Prakash J, G. Bharath, S. Anwer, P. Veluswamy, D. Prema, K. S. Venkataprasanna, and G. D. Venkatasubbu. "Investigations on the in-vivo toxicity analysis of reduced graphene oxide/TiO2 nanocomposite in zebrafish embryo and larvae (Danio rerio)." Applied Surface Science 481, no. (2019): 1360-1369. doi:10.1016/j.apsusc.2019.03.287. 
[146] Younes, N., G. Pintus, M. Al-Asmakh, K. Rasool, S. Younes, S. Calzolari, K. A. Mahmoud, and G. K. Nasrallah. "“Safe” Chitosan/Zinc Oxide Nanocomposite Has Minimal Organ-Specific Toxicity in Early Stages of Zebrafish Development." ACS Biomaterials Science \& Engineering no. (2019). doi: 10.1021/acsbiomaterials.8b01144.

[147] Zheng, M., J. Lu, and D. Zhao. "Effects of starch-coating of magnetite nanoparticles on cellular uptake, toxicity and gene expression profiles in adult zebrafish." Science of The Total Environment 622-623, no. (2018): 930-941. doi:10.1016/j.scitotenv.2017.12.018.

[148] Da Silva, G. H., Z. Clemente, L. U. Khan, F. Coa, L. L. R. Neto, H. W. P. Carvalho, V. L. Castro, D. S. T. Martinez, and R. T. R. Monteiro. "Toxicity assessment of TiO2-MWCNT nanohybrid material with enhanced photocatalytic activity on Danio rerio (Zebrafish) embryos." Ecotoxicology and Environmental Safety 165, no. (2018): 136-143. doi: 10.1016/j.ecoenv.2018.08.093.

[149] Gedda, G., Y.-Y. Yao, S.-H. Chen, A. V. Ghule, Y.-C. Ling, and J.-Y. Chang. "Facile synthesis of gold/gadolinium-doped carbon quantum dot nanocomposites for magnetic resonance imaging and photothermal ablation therapy." Journal of Materials Chemistry B 5, no. 31 (2017): 6282-6291. doi: 10.1039/C7TB01139C.

[150] Kandiah, K., R. Venkatachalam, C. Wang, S. Valiyaveettil, and K. Ganesan. "In vitro and preliminary in vivo toxicity screening of high-surface-area TiO2-chondroitin-4-sulfate nanocomposites for bone regeneration application." Colloids and Surfaces B: Biointerfaces 128, no. (2015): 347-356. doi: 10.1016/j.colsurfb.2015.02.027.

[151] Poureetezadi, Shahram Jevin, and Rebecca A. Wingert. "Little Fish, Big Catch: Zebrafish as a Model for Kidney Disease." Kidney International 89, no. 6 (June 2016): 1204-1210. doi:10.1016/j.kint.2016.01.031.

[152] Barriuso, J., R. Nagaraju, and A. Hurlstone. "Zebrafish: A New Companion for Translational Research in Oncology." Clinical Cancer Research 21, no. 5 (2015): 969. doi: 10.1158/1078-0432.CCR-14-2921.

[153] Morningstar Jordan, E., K. Nath Anjali, F. O'Sullivan John, B. Zheng, T. Peterson Randall, and E. Gerszten Robert. "Abstract 19501: DMGV is Predictive of Future Diabetes, and Modulates Glucose Homeostasis in Zebrafish." Circulation 136, no. suppl_1 (2017): A19501-A19501. doi: 10.1161/circ.136.suppl_1.19501.

[154] Sieg, R. E., E. S. Hausman, and J. Blackburn. "Abstract 3019: PRL3 contributes to the onset and progression of T-cell acute lymphoblastic leukemia in a zebrafish model." Cancer Research 78, no. 13 Supplement (2018): 3019. doi: 10.1158/15387445.AM2018-3019.

[155] Keller, Jill M., and Evan T. Keller. "The Use of Mature Zebrafish ( Danio Rerio ) as a Model for Human Aging and Disease." Conn's Handbook of Models for Human Aging (2018): 351-359. doi:10.1016/b978-0-12-811353-0.00026-9.

[156] Braunbeck, T., B. Kais, E. Lammer, J. Otte, K. Schneider, D. Stengel, and R. Strecker. "The fish embryo test (FET): origin, applications, and future." Environmental Science and Pollution Research 22, no. 21 (2015): 16247-16261. doi:10.1007/s11356014-3814-7. 